\title{
Epidemiological survey of Microsporum canis infection diagnosed at Nagasaki University Hospital for 20 years (1996-2015)
}

\author{
Motoi Takenaka (Nagasaki Univesity Hospital, Department of dermatoallergology), \\ Katsutaro Nishimoto (Japan seafarers relief association Nagasaki hospital)
}

\section{【Introduction】}

In Japan, the Microsporum (M.) canis infection had been reported since 1990's as a causative fungus species of an increasing tinea capitis (T. capitis) and tinea corporis ( $T$. corporis) patients. On the other hand, since 2001 in Japan, Trichophyton $(T$.$) tonsurans infections ( T$. capitis and $T$. corporis) were become reported increasing especially among the high school students related to contact sports playing, in contrast to the remarkable decrease of M.canis infection. To clarify the present situation, we surveyed the trend of M.canis infectious in Nagasaki area.

\section{【Material and Method】 \\ No. of Cases

$$
\begin{aligned}
& 1996 \sim 2000: 10177 \text { cases } \\
& 2001 \sim 2005: 10347 \text { cases } \\
& 2006 \sim 2010: 7573 \text { cases } \\
& 2011 \sim 2015: 3244 \text { cases }
\end{aligned}
$$

The clinical samples that gathered from tinea patients had been send mainly by dermatologists of Nagasaki area. Identification of dermatophytes was made by macro- and micro- morphology of primary isolates and for the difficult strains, the molecular biological examination.

\section{【Results】}

M.canis was identified in 196 cases (1.2\%) from 1996 to 2000,123 cases $(1.2 \%)$ form 2001 to 2005,145 cases $(1.9 \%)$ from 2006 to 2010, 170 cases (5.2\%) from 2011 to 2015, showing the isolation rate of M.canis tend to increase.
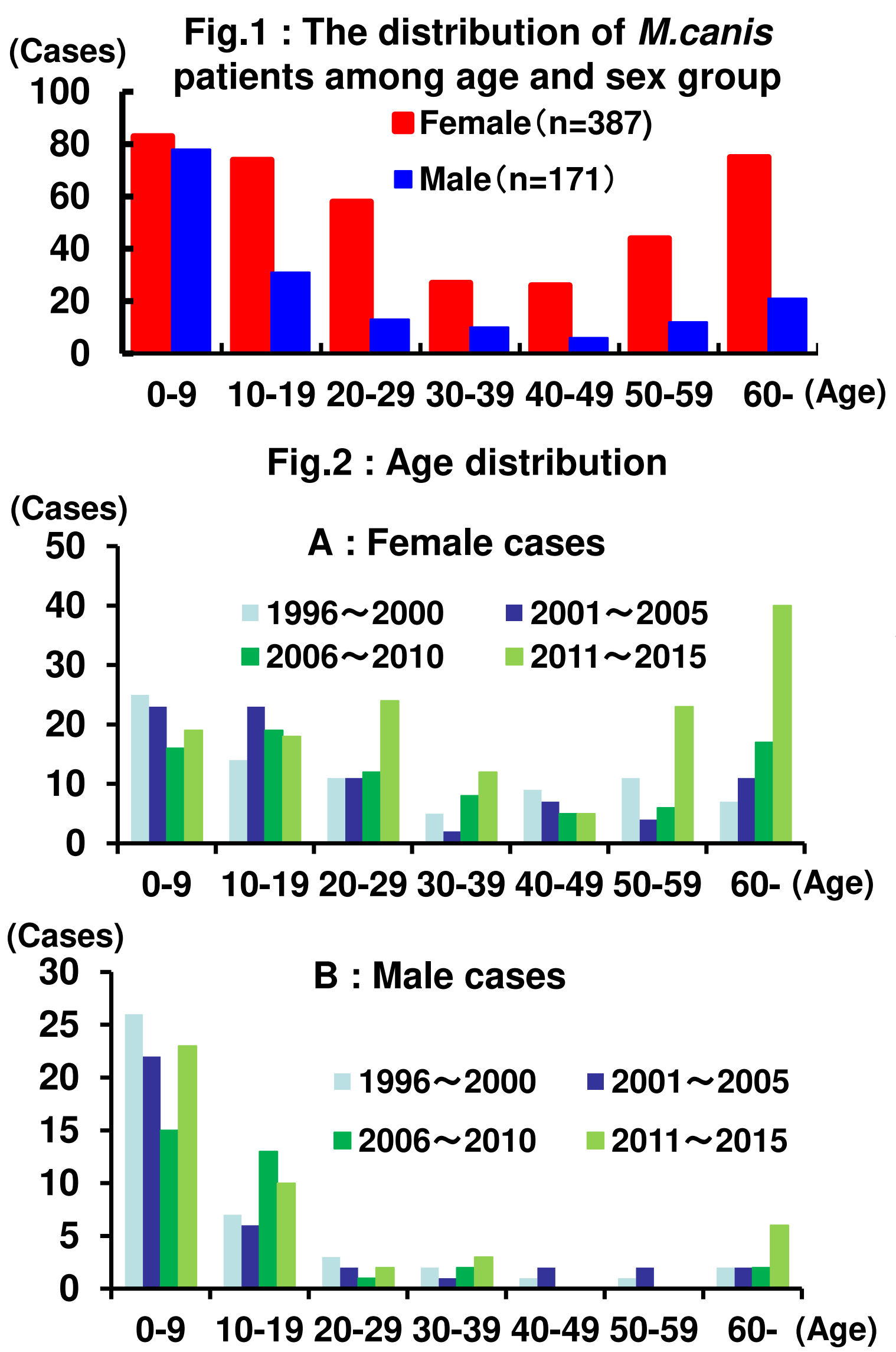

The distribution among age groups was showed in Fig.1. With female patients (total of 387 cases), under 19 years old (0-9 years old: 83 cases, 10-19 years old: 74 cases) and over 60 years old (75cases) accounted for the most. Recently the increase of female cases of over 60 years old was conspicuous (Fig.2-A).

With male patients (total of 156 cases), under 9 years old (86 cases) were affected most. This tendency did not change for recently 20 years (Fig.2-B).

Transmission from animals was examined and 157 cases in $634(24.8 \%)$ were confirmed to relate with pet animals, especially cats. With the cases of familial occurrence, the rate increased up to $60.4 \%$.

In Japan, the infestation of T.tonsurans was increasing since recent 20 years, especially from 2006 in Nagasaki. Therefor it became important to know the causative fungus species of tinea lesions for the effective control of infection souses. In the patients over 20-year-old (Fig.4-A), T.rubrum occupied the most part of the causative species in $\mathrm{T}$. corporis. It tended to be similar even in tinea faciei (T. faciei) and T. capitis. On the other hand, $T$. tonsurans was the most mainly identified even in either disease types in the patient of 19-year-old or less (Fig.4-B). However, M.canis occupied 10 20\% in either age groups and types of tinea lesion.

\section{【Conclusion】}

M.canis is proved still an important dermatophyte species in Nagasaki prefecture among children and aged females suggesting their frequent contact with pet animals.

Fig. 3 : The relation with the pet animal
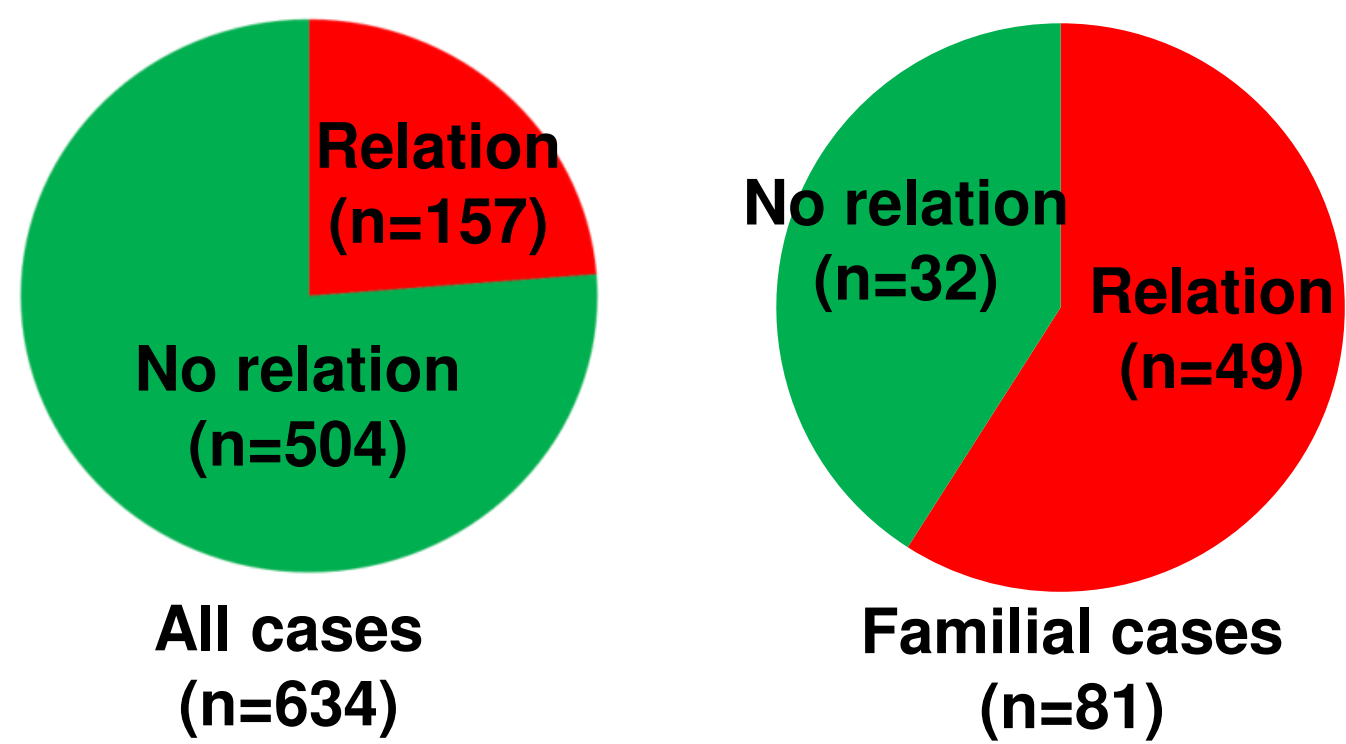

Fig.4 : Identified fungus species by disease types from 2006 to 2015

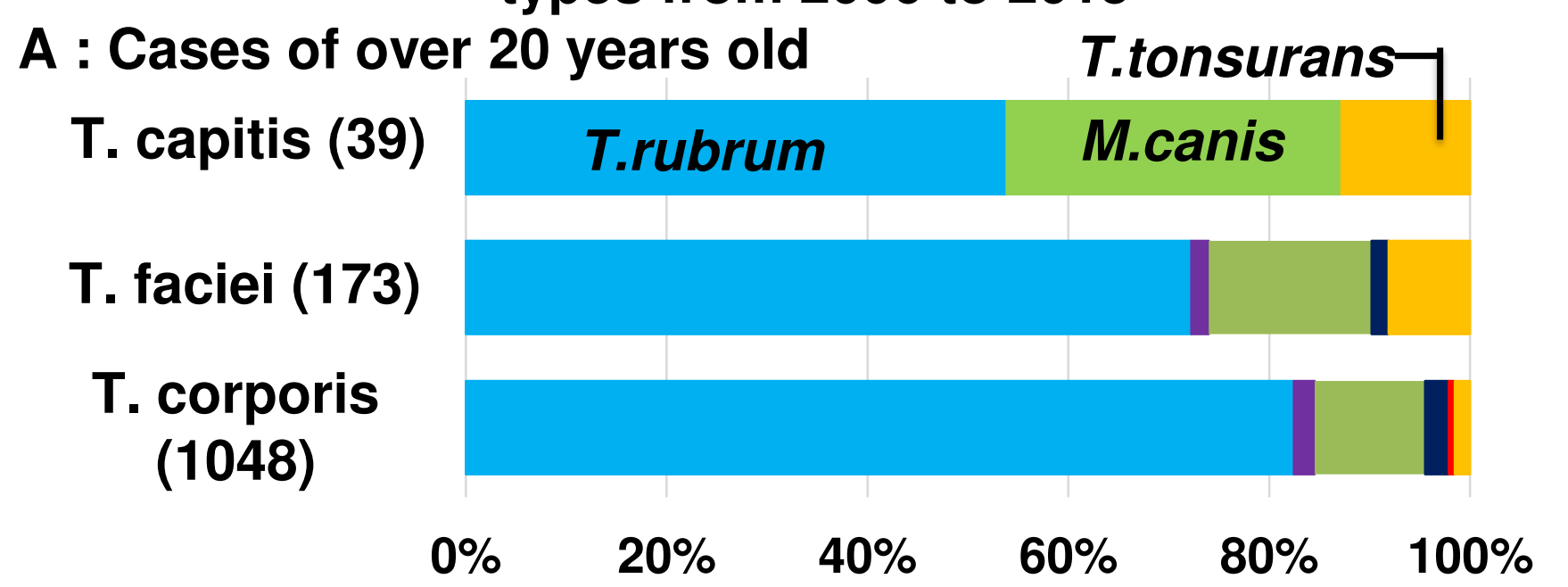

$B$ : Cases of under 19 years old

T. capitis (191) M.canis T.tonsurans

T. faciei (157)

T. corporis T.rubrum 